\title{
In-Ear Pulse Wave Measurements: A Pilot Study
}

\author{
Steffen Kaufmann, Gunther Ardelt, Ankit Malhotra, Martin Ryschka \\ Laboratory for Medical Electronics, Lübeck University of Applied Sciences, Germany
}

ryschka@fh-luebeck.de

\begin{abstract}
The measurement of the Pulse wave Arrival Time (PAT) has proven to be a vital tool in medical diagnosis. Whereby most PAT measurements are carried out at extremities, this work proposes the interior of the ear as a new site. Due to pressure variations inside the auditory canal a pulse wave can be measured by using a pressure sensor or by simple in-ear headphones. To verify the signal origin, a reflectance photoplethysmograph (PPG) measurement inside the ear is carried out. All sensors are integrated for accurate and comfortable fit, in a custom made mould.
\end{abstract}

Keywords: Auditory canal, in-ear headphones, in-ear pressure, $P P G, E C G, P A T$

\section{Introduction}

A key to future patient monitoring is portability and adaptability not only to hospital setups, but also to various other environments such as home. For that purpose it is desirable to record vital signs non-invasively and comfortably for a long period of time.

Pulse wave Arrival Time (PAT) and pulse wave morphology are considered as indicators of arterial stiffness and are also known as (prognostic) markers for cardiac and vascular diseases $[1,2]$. In the past various methods and sites have been demonstrated for pulse wave measurements, mostly on carotid and femoral artery through invasive and non-invasive methods.

This work proposes the auditory canal as a new site for pulse wave detection and PAT measurements. For that purpose the auditory canal is sealed and the internal pressure (in-ear pressure) variation is detected. For providing a time reference, a reflective photoplethysmography (PPG) sensor is placed inside the canal and a simultaneous ECG recording is carried out, additionally.

\section{Materials and Methods}

For non-invasive vital signs monitoring the auditory canal is appropriate in both anatomical and physiological aspects. Anatomically the auditory canal is located in the temporal bone, whereby it is partially composed of cartilage and bone. Its length is approximately $2.5 \mathrm{~cm}$, while the diameter is about $0.7 \mathrm{~cm}$. The ears remain at the same orientation relative to the heart, while the subject is in an upright position, e. g. this reduces the need for complex calibrations when calculating Pulse Wave Velocity (PWV) [3]. Furthermore the auditory canal is a superior site in terms of patient's acceptance and also a good anchoring point for the device. Various other studies also propose the position behind the ear as a suitable location for vital sign monitoring, in regard to reduced motion artifacts $[4,5]$.

Physiologically the auditory canal is richly perfused via various subdivisions of internal and external carotid arteries. The volume change of the elastic vessels couples into the auditory canal, hence when the canal is sealed a pressure variation can be measured.

Measurement setup: For the pressure measurement two different sensors are evaluated: a normal commercial inear headphone and a low-range differential pressure sensor. For comparison a reflective PPG is recorded inside the ear, for providing a time reference additionally an ECG is recorded. To allow further analysis, the measured waveforms are digitized via an Analog to Digital Converter (ADC) and transmitted to a host PC. Figure 1 shows the used measurement setup.

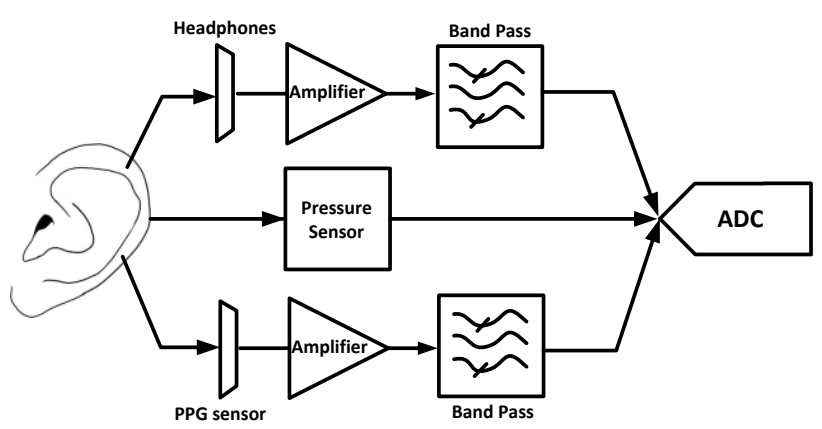

Figure 1: Block diagram of the in-ear measurement setup headphones and pressure sensor are not located in the same mould; ECG is not shown.

Because of size limitations of the experimental setup the in-ear headphone is used in the right ear. The pressure sensor, together with the reflective PPG sensor, is integrated into a custom made mould, which is applied to the left ear.

Headphones: Commercial in-ear headphones are used to detect the in-ear pressure variation. The signals from the headphones are amplified and band pass filtered and then fed to Analog to Digital Converter (ADC). The band pass filter has cut of frequencies of $16 \mathrm{mHz}$ and $30 \mathrm{~Hz}$, respectively. As the headphones are used below their intended frequency range the exact trend of its transfer function is unknown and the waveform shape may be deformed. To compensate the inductive behavior of the headphone, its signal needs to be integrated.

Pressure measurement: The setup consists of a differential pressure sensor (HCEM010DBE85P, First Sensor GmbH) 
with a pressure range of $\pm 1000 \mathrm{kPa}$ ( \pm 10 mbar). The pressure sensor is connected via a small hose to the mould. It is very lightweight and small sized therefore it can be mounted in front of the ear.

PPG measurement: The in-ear site is well-suited for PPG measurements, because of the absence of ambient light. The used fundamental setup consists of a $940 \mathrm{~nm}$ infrared sensor and a photodiode with corresponding wavelength sensitivity. The output signal is amplified and band pass filtered before ADC. The cut off frequencies are $16 \mathrm{mHz}$ and $30 \mathrm{~Hz}$, respectively.

Sensor mould for inside ear measurements: To sense the pressure signal together with the PPG signal inside the ear a hybrid sensor is developed, which can be easily fits into the small auditory canal. For this purpose an autoplastic silicone based compound is used. The compound is an optically clear, non-toxic, non-inflammable flexible material, well known for auditory prosthesis applications. Figure 2 shows the ear mould with the two different sensors attached.

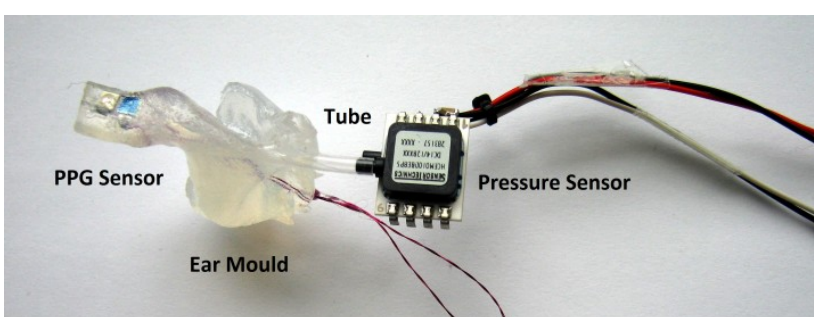

Figure 2: The hybrid sensor consisting of in-ear pressure and PPG sensors.

For the manufacturing of the particular mould, silicone is injected via a gun into the auditory canal and after a period of about 7 minutes the material automatically hardens. Afterwards the sensors are attached to the mould. The advantage is easy fitting and sealing of the auditory canal from the ambient environment.

\section{Results}

The analysis of the recorded waveforms is done with MathWorks MATLAB. To enhance the signal quality zero-phase band-pass filters are employed. Figure 3 shows the filtered and normalized waveforms.

Because of the unknown transfer function of the headphone sensor absolute pressure values are omitted. The pressure variation measured with the pressure sensor was found to be about $15 \mathrm{~Pa}$.

It can be observed that the onset of pressure and PPG signals are synchronous, but delayed to the R-peak of ECG. The PAT derived from all three measured in-ear pulse waveforms are about $100 \mathrm{~ms}$, whereby the PAT on extremities like ankle or fingertip is usually reported in the range of 244-267 ms [6].

\section{Summary and Outlook}

It is shown that pulse waveforms can be derived from in-ear PPG and pressure measurements. It is also shown that commercial in-ear headphones can be used as pulse waveform sensors. Furthermore PAT values can be derived from those waveforms.

As the origin of the pressure variation is still a hypothesis, further research is necessary to investigate its genesis. The morphology of the waveform derived from the in-ear headphone is still under question and further work is needed to quantify the effect of its transfer function.

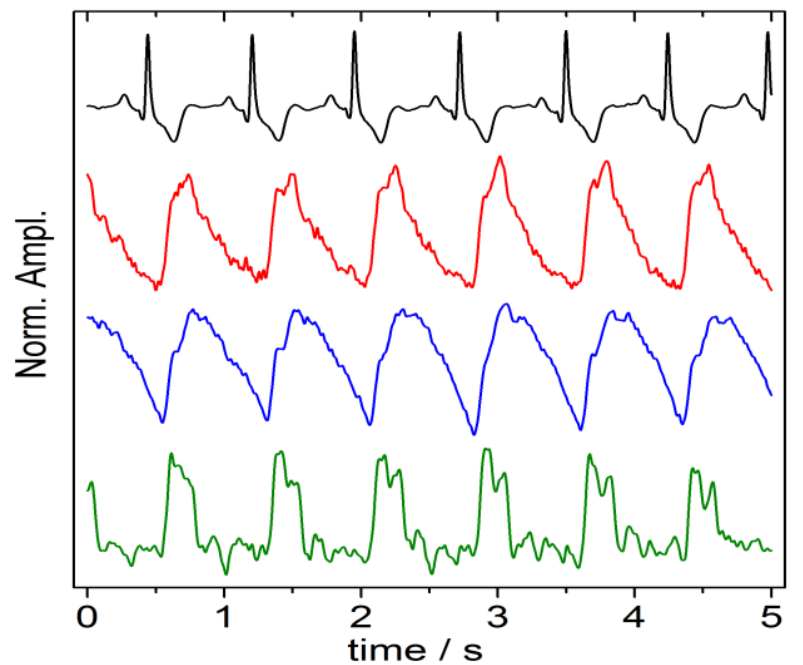

Figure 3: The measured waveforms from the in-ear sensors (from top to bottom) - ECG, in-ear PPG (left ear), in-ear pressure measured with pressure sensor (left ear) and headphone (right-ear)

\section{Acknowledgement}

This work was supported by the German Federal Ministry of Education and Research under the grant number 13EZ1140B.

\section{Bibliography}

[1] Laurent S., Boutouyrie P., Asmar R., et al: Aortic stiffness is an independent predictor of all-cause and cardiovascular mortality in hypertensive patients. Hypertension 2001; 37: p. 1236-1241

[2] Guerin A. P., Blacher J, Pannier B, Marchais SJ, Safar ME, London GM: Impact of aortic stiffness attenuation on survival of patients in end-stage renal failure. Circulation 2001; 103: p. 987-992

[3] Guo D. G., Tay F. E. H., Xu L., et al., "A long-term wearable vital signs monitoring system using BSN," IEEE Euromico Conf. on Dig.Sys. Des. Arch, Italy, Sept. 2008, pp. 825-830

[4] He, D. D, Winokur E. S., Heldt, T., Sodini C. G.: " The Ear as a Location for Wearable Vital Signs Monitoring. In: Proceedings of the 32nd Annual International Conference of the IEEE EMBS, 2010, p. 6389-6392

[5] Winokur E.S., He, D. D.; Sodini C. G.: A Wearable Vital Signs Monitor at the Ear for Continuous Heart Rate and Pulse Transit Time Measurements. In: e IEEE EMBS2012

[6] M C Kortekaas et al.: Comparison of bilateral pulse arrival time before and after induced vasodilation by axillary block. Physiol. Meas. 2012,33, p.1993-2002 